\title{
Effortful control, depression, and anxiety correlate with the influence of emotion on executive attentional control
}

\author{
Philipp Kanske ${ }^{a, *}$, Sonja A. Kotz ${ }^{b}$ \\ a Max Planck Institute for Human Cognitive and Brain Sciences, Department of Social Neuroscience, Leipzig, Germany \\ ${ }^{\mathrm{b}}$ Max Planck Institute for Human Cognitive and Brain Sciences, Neurocognition of Rhythm in Communication Group, Leipzig, Germany
}

\section{A R T I C L E I N F O}

\section{Article history:}

Received 28 June 2011

Accepted 26 April 2012

Available online 5 May 2012

\section{Keywords:}

Temperament

Affect

Conflict

Anterior cingulate cortex

Amygdala

Simon task

Flanker task

\begin{abstract}
A B S T R A C T
Recent evidence confirms that emotion can trigger executive attentional control. Participants resolve conflict faster when encountering emotionally negative or positive stimuli. This effect is accompanied by an enlarged conflict negativity in event-related brain potentials (ERPs) and activation of the ventral anterior cingulate cortex (ACC) in fMRI. Here, we tested whether temperament (the trait effortful control) and subclinical factors (anxiety, depression) can influence the emotional modulation of executive attention. These factors correlated with conflict processing in six experiments that utilized different conflict tasks (flanker, Simon) and different types of emotional stimuli (visual, auditory). Participants high in effortful control and low in anxiety and depression responded faster to conflict processing in emotional stimuli, showed an enhanced ERP conflict negativity, and additional activation in the ventral ACC. The data show that temperamental effortful control, depression, and anxiety are related to the influence of emotion on executive attention and its underlying neural correlates.
\end{abstract}

(C) 2012 Elsevier B.V. All rights reserved.

\section{Introduction}

It has long been hypothesized that emotion may influence executive control of attention. For example, Norman and Shallice (1986) argued that a number of different situations trigger attentional control, including errors, novelty and also danger is signaled by emotional stimuli. Several recent studies show that emotion can indeed speed up attentional control processes. Using different conflict tasks as a measure of attentional control, we reported faster resolution of conflict when target stimuli were emotional compared to neutral stimuli (Kanske and Kotz, 2010b, 2011a,c,d,e). This adaptive mechanism reduces the time that conflicting action tendencies yield an organism unable to react in highly salient situations such as those signaled by emotion. This mechanism is subserved by a neural network comprising the ventral and dorsal portions of the anterior cingulate cortex (ACC), as well as the amygdala (Kanske and Kotz, 2011c,d). While the dorsal ACC is involved in conflict processing irrespective of emotion, the ventral ACC is recruited for conflict processing only when target stimuli are emotional, potentially via input from the amygdala as indicated by increased functional connectivity. Recordings from the electroencephalogram indicate the rapid speed of this mechanism,

\footnotetext{
* Corresponding author at: Max Planck Institute for Human Cognitive and Brain Sciences, Department of Social Neuroscience, Stephanstr. 1a, 04103 Leipzig, Germany. Tel.: +49 3419940 2693; fax: +49 34199402356.

E-mail address: kanske@cbs.mpg.de (P. Kanske).
}

as emotion already modulates the first stages of conflict processing in event-related brain potentials (ERP; Kanske and Kotz, 2010b, 2011a,e).

Temperament-related differences in effortful control have been shown to relate to attentional control (Rueda et al., 2005), and individuals' emotional states modulate the perception of emotional stimuli (Li et al., 2008). Therefore, they may also relate to the influence of emotion on attentional control. This is a critical question, which could yield insight into the factors that influence this relation and explain interindividual variability. To probe this question, we applied a correlational approach and tested whether individual differences in effortful control and subclinical depression and anxiety correlate with the speeding up of conflict processing and its underlying neural mechanisms.

Temperamental effortful control describes an individual's capacity for self-regulation (Rothbart, 2007). More specifically, it has been defined as the "ability to choose a course of action under conditions of conflict, to plan for the future, and to detect errors" (Rothbart, 2007, p. 207). The concept has mainly been applied in developmental research involving psychometric and laboratory studies (Rothbart and Bates, 2006). Nevertheless, effortful control can also be reliably measured in adults using the adult temperament questionnaire, which was used in the present study (ATQ Evans and Rothbart, 2007; Rothbart et al., 2000).

The relation of effortful control and executive attention has been extensively studied in children. Children high in effortful control commonly exhibit better performance in conflict tasks (Gerardi-Caulton, 2000; Rothbart et al., 2003). Evidence of such a 
relation also comes from biopsychological data. Both effortful control (Goldsmith et al., 1999) and executive attention (Fan et al., 2001) have been shown to be heritable. For example, Rueda et al. (2005) investigated the dopamine-related DAT1 gene which is, among other dopamine genes, related to executive attention (Blasi et al., 2005; Diamond et al., 2004; Fan et al., 2003; Fossella et al., 2002). They found that the long form of the DAT1 gene was associated with higher effortful control, better conflict scores, and a reliable ERP conflict negativity, which children with the mixed allele did not show. ${ }^{1}$ This strong evidence suggests that temperamental effortful control can explain inter-individual differences in executive attention. However, it is unclear whether this relation is also present in adults. Furthermore, it is unknown whether effortful control affects the influence of emotion on executive attentional control.

Interestingly, there is some data that hint at a potential role of effortful control in emotion processing. Kochanska et al. (2000) found that effortful control measured with a battery of simple tasks at 22 months of age was associated with the ability to regulate anger, and predicted anger and joy regulation at 33 months of age. A positive relation of effortful control to emotion regulation has also been reported by several other groups (Carlson and Wang, 2007; Salmon and Pereira, 2002; Valiente et al., 2007, for a review, see Spinrad et al., 2007). In addition, effortful control is related to several other measures including delay of gratification, the development of conscience, the development of empathy, and lower levels of psychopathology and maladjustment (Eisenberg, 2000; Kochanska, 1997; Kochanska et al., 2000; Krueger et al., 1996; White et al., 1994). Effortful control has even been found to negatively correlate with subclinical depression and anxiety (Moriya and Tanno, 2008).

Emotional states such as depression and anxiety strongly modulate the perception of emotional stimuli. This has been observed in patients with specific phobias. Spider phobics, for example, detect and will be distracted by spiders much faster and stronger than nonphobic participants (Gerdes et al., 2008; Mühlberger et al., 2006). Furthermore, generalized anxiety disorder (Bradley et al., 1999) and depression (Mitterschiffthaler et al., 2008) mainly affect the processing of negative emotional stimuli. Interestingly, these effects can be observed not only in clinical, but also in subclinical anxiety and depression, i.e., different anxiety levels in healthy participants can explain differences in processing of emotional stimuli (Li et al., 2008; Mercado et al., 2006; Scott et al., 2001). The neural basis of the heightened sensitivity to emotional stimuli in anxiety and depression includes altered processing in emotion-relevant brain regions such as the amygdala and the ventral ACC. In depression, amygdala activation to negative emotional stimuli is increased for pictures (Lee et al., 2007) and words (Siegle et al., 2007). This effect is even present when the presentation of negative but not positive emotional stimuli is anticipated, but no stimulus is presented (Abler et al., 2007). In contrast, activation of the ventral ACC can be reduced in depression (Drevets et al., 1997). Volume changes in the amygdala and ventral ACC in depression have also been observed (Pezawas et al., 2005; for reviews about the neural basis of depression, see Drevets, 2001; Kalia, 2005). Similar effects have been reported in anxiety, also regarding the amygdala response (Bishop et al., 2004; Most et al., 2006; for a review, see Bishop, 2007). This influence of depression and anxiety on emotion processing occurs already very early after stimulus onset as documented in early ERP

\footnotetext{
1 An ERP negativity has consistently been observed in conflict tasks. The timing of this negativity, however, has varied in different conflict tasks. Accordingly, the component has been labeled differently (Folstein and Van Petten, 2008). As we applied two different conflict tasks in the experiments reported here, we use the term "ERP conflict negativity" throughout the manuscript to avoid confusion.
}

effects (e.g., P1) and reactions to subliminally presented pictures (Mercado et al., 2006), faces (Bar-Haim et al., 2005) and words (Li et al., 2007, 2008; Weinstein, 1995). There are also several studies yielding insight into the nature of these differences. Participants scoring high in anxiety and depression seem to be faster to detect negative emotional stimuli, orient attention to stimuli, and experience difficulties in disengaging from them (Bradley et al., 1999; Fox et al., 2001, 2002; Garner et al., 2006; Mogg and Bradley, 1999; Mogg et al., 2000, 2004, 2007; for a meta-analysis, see Bar-Haim et al., 2007).

In summary, temperamental effortful control is associated with efficient conflict processing, but also the regulation of emotion. Altered processing of emotional stimuli in anxiety and depression indicates that the relevance of a stimulus in a given situation may not be appropriately detected. This fits well with the pattern of hyper- and hypoactivation in amygdala and ACC.

Thus, heightened sensitivity to negative stimuli in anxiety and depression, and the negative correlation of regulatory processing (effortful control) and depression and anxiety suggest less appropriate evaluation of emotional stimuli. Consequently, the supportive role of emotion in guiding executive control of attention could be affected by inter-individual differences in effortful control, depression, and anxiety. Therefore, we assessed effortful control, depression, and anxiety through questionnaires and correlated them to the speed of conflict processing in emotional trials. We analyzed this potential impact in six previously published experiments based on different conflict tasks (flanker and Simon type of conflict) and stimuli in different sensory modalities (visual and auditory). We hypothesized that participants high on depression and anxiety, but low on effortful control scores would show a reduced benefit from emotion in conflict resolution response times. Furthermore, we expected to find a relation to the underlying neural mechanisms measured with ERPs and functional magnetic resonance imaging (fMRI). As the ERP conflict negativity is increased in emotional trials with better behavioral conflict resolution, the amplitude increase should be smaller in high depression, anxiety and low effortful control. Similarly, activation increase of the ventral ACC has been related to enhanced conflict resolution and should, therefore, be reduced in high depression, anxiety and low effortful control.

\section{Materials and methods}

\subsection{Participants}

A total of 142 participants ( 70 females) were included in the analysis. They were distributed across six separate experiments (see Table 1 for details). Five participants were excluded because of excessive EEG artifacts. The mean age was 24.2 (SD 2.3), there were no significant age differences between the six subgroups $(p>30)$. All participants were native speakers of German, right-handed according to the Edinburgh Handedness Inventory (Oldfield, 1971), with a mean laterality quotient of 95.7 (SD 6.9). Participants reported normal or corrected-to-normal vision and normal hearing and no history of neurological or psychiatric disorders as assessed in a screening questionnaire that asked for previous diagnoses and treatments. The study was approved by the Ethics Committee of the University of Leipzig. All participants gave written informed consent prior to participation.

\subsection{Questionnaires}

\subsubsection{Depression Anxiety Stress Scales (DASS)}

To assess the subclinical range of depression and anxiety, the DASS (Lovibond and Lovibond, 1995b) were administered. In addition to depression and anxiety, this questionnaire also measures stress levels, each scale consists of 14 items. In the instruction, participants are asked to evaluate whether an item applied to them during the last week. There were four response options ranging from "did not apply to me at all" to "applied to me very much, or most of the time. Scores of 0-3 were assigned to each response option and mean scores for each participant and scale were calculated. Reliability (Cronbach's alpha) in nonclinical samples is for the depression scale .91 , the anxiety scale .84 , for the stress scale .90 (Lovibond and Lovibond, 1995b). Cronbach's alpha for the present samples is reported in Table 2. Example items are: "I felt that I had nothing to look forward to." "I felt I was pretty 
Table 1

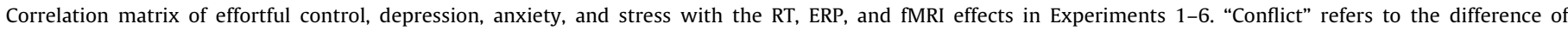

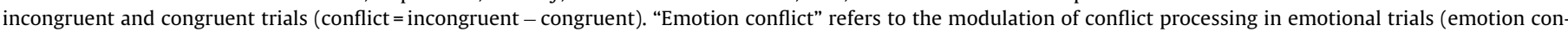

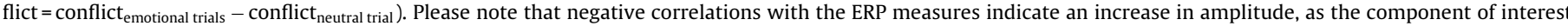
was a negative deflection.

\begin{tabular}{|c|c|c|c|c|c|c|}
\hline & & & Effortful control & Depression & Anxiety & Stress \\
\hline $\begin{array}{l}\text { Experiment } 1 \\
\text { Visual flanker task } \\
\text { Negative emotion } \\
N=26 \text { ( } 14 \text { females) }\end{array}$ & $\begin{array}{l}\text { RT } \\
\text { ERP negativity }\end{array}$ & $\begin{array}{l}\text { Conflict } \\
\text { Emotion conflict } \\
\text { Conflict } \\
\text { Emotion conflict }\end{array}$ & $\begin{array}{l}-.49^{*} \\
-.39^{*} \\
-.40^{*} \\
-.43^{*}\end{array}$ & $\begin{array}{c}-.04 \\
.57^{* *} \\
-.04 \\
.66^{*}\end{array}$ & $\begin{array}{c}.12 \\
.62^{* *} \\
-.03 \\
.48^{*}\end{array}$ & $\begin{array}{r}.11 \\
.32 \\
.01 \\
-.15\end{array}$ \\
\hline $\begin{array}{l}\text { Experiment } 2 \\
\text { Visual flanker task } \\
\text { Positive emotion } \\
N=25 \text { (12 females) }\end{array}$ & $\begin{array}{l}\text { RT } \\
\text { ERP negativity }\end{array}$ & $\begin{array}{l}\text { Conflict } \\
\text { Emotion conflict } \\
\text { Conflict } \\
\text { Emotion conflict }\end{array}$ & $\begin{array}{l}-.41^{*} \\
-.43^{*} \\
-.46^{*} \\
-.48^{*}\end{array}$ & $\begin{array}{r}.16 \\
-.13 \\
.17 \\
-.25\end{array}$ & $\begin{array}{r}.11 \\
-.17 \\
.00 \\
-.29\end{array}$ & $\begin{array}{r}-.04 \\
-.25 \\
.05 \\
.02\end{array}$ \\
\hline $\begin{array}{l}\text { Experiment } 3 \\
\text { Visual flanker task } \\
\text { Negative emotion } \\
N=20 \text { (10 females) }\end{array}$ & $\begin{array}{l}\text { RT } \\
\text { L vACC } \\
\text { R vACC } \\
\text { L amygdala } \\
\text { R amygdala }\end{array}$ & $\begin{array}{l}\text { Conflict } \\
\text { Emotion conflict } \\
\text { Emotion conflict } \\
\text { Emotion conflict } \\
\text { Emotion } \\
\text { Emotion }\end{array}$ & $\begin{array}{c}-.43^{\#} \\
-.45^{*} \\
.45^{*} \\
.45^{*} \\
.31 \\
.12\end{array}$ & $\begin{array}{r}-.13 \\
.45^{*} \\
-.47^{*} \\
-.52^{*} \\
.46^{*} \\
.49^{*}\end{array}$ & $\begin{array}{r}-.16 \\
.47^{*} \\
-.45^{*} \\
-.49 \\
.46^{*} \\
.51^{*}\end{array}$ & $\begin{array}{r}.19 \\
-.20 \\
-.16 \\
-.15 \\
.22 \\
-.17\end{array}$ \\
\hline $\begin{array}{l}\text { Experiment } 4 \\
\text { Auditory Simon task } \\
\text { Negative emotion } \\
N=23 \text { (10 females) }\end{array}$ & $\begin{array}{l}\text { RT } \\
\text { ERP negativity }\end{array}$ & $\begin{array}{l}\text { Conflict } \\
\text { Emotion conflict } \\
\text { Conflict } \\
\text { Emotion conflict }\end{array}$ & $\begin{array}{l}-.56^{* *} \\
-.47^{*} \\
-.49^{*} \\
-.45^{*}\end{array}$ & $\begin{array}{l}.00 \\
-.66^{* *} \\
-.14 \\
.65^{* *}\end{array}$ & $\begin{array}{l}.00 \\
-.76^{* *} \\
-.12 \\
-.53^{* *}\end{array}$ & $\begin{array}{r}-.13 \\
.10 \\
-.04 \\
.15\end{array}$ \\
\hline $\begin{array}{l}\text { Experiment } 5 \\
\text { Auditory Simon task } \\
\text { Positive emotion } \\
N=26 \text { ( } 14 \text { females) }\end{array}$ & $\begin{array}{l}\text { RT } \\
\text { ERP negativity }\end{array}$ & $\begin{array}{l}\text { Conflict } \\
\text { Emotion conflict } \\
\text { Conflict } \\
\text { Emotion conflict }\end{array}$ & $\begin{array}{l}-.46^{*} \\
-.42^{*} \\
-.47^{*} \\
-.41^{*}\end{array}$ & $\begin{array}{r}-.04 \\
.28 \\
.02 \\
.19\end{array}$ & $\begin{array}{r}.21 \\
.23 \\
.18 \\
-.10\end{array}$ & $\begin{array}{r}-.21 \\
.22 \\
.36 \\
-.06\end{array}$ \\
\hline $\begin{array}{l}\text { Experiment } 6 \\
\text { Auditory Simon task } \\
\text { Negative emotion } \\
N=22(10 \text { females })\end{array}$ & $\begin{array}{l}\text { RT } \\
\text { R vACC } \\
\text { L amygdala } \\
\text { R amygdala }\end{array}$ & $\begin{array}{l}\text { Conflict } \\
\text { Emotion conflict } \\
\text { Emotion conflict } \\
\text { Emotion } \\
\text { Emotion }\end{array}$ & $\begin{array}{r}-.44^{*} \\
-.53^{*} \\
.51^{*} \\
.21 \\
-.04\end{array}$ & $\begin{array}{c}.33 \\
.44^{*} \\
-.43^{*} \\
.38^{\#} \\
.46^{*}\end{array}$ & $\begin{array}{c}.33 \\
.43^{*} \\
-.44^{*} \\
.37^{\#} \\
.45^{*}\end{array}$ & $\begin{array}{r}.02 \\
.12 \\
-.23 \\
-.35 \\
-.30\end{array}$ \\
\hline
\end{tabular}

** $p<.05$

\# $p<.10$.

worthless." (depression); "I felt scared without any good reason." "I felt that I would be 'thrown' by some trivial, but unfamiliar task." (anxiety); "I found it hard to calm down after something upset me." "I found it difficult to relax." (stress). Normative data in a large non-clinical student sample $(N=717,486$ females, mean age $=21.0$ years) have been reported (Lovibond and Lovibond, 1995a) with a sum score of $7.19(\mathrm{SD}=6.54)$ for depression, $5.23(\mathrm{SD}=4.83)$ for anxiety, and $10.54(\mathrm{SD}=6.94)$ for stress. All participants were in the range of normal to moderate scores in the scales (i.e., 0-20 for depression, 0-14 for anxiety, and 0-25 for stress).

\subsubsection{Adult Temperament Questionnaire (ATQ)}

We also measured effortful control with the respective scale taken from the Adult Temperament Questionnaire (ATQ), which has a reliability of .75 (Rothbart et al., 2000). Cronbach's alpha for the present samples is reported in Table 2. The scale consists of 35 items and includes three sub-constructs; inhibitory control, activation control, and attentional control. Because the general concept of effortful control was of interest in the present study, the sub-constructs were not analyzed separately. Participants were instructed to judge how well an item described them. There were seven response options ranging from "extremely untrue" to "extremely true", with the additional option "not applicable". Scores of 1-7 were assigned to each response option and mean scores for each participant were calculated. Example items from the questionnaire are: "I usually finish doing things before they are actually due." "When I see an attractive item in a store, it's usually very hard for me to resist buying it." "When trying to study something, I have difficulty tuning out background noise and concentrating." Normative data for effortful control have been reported (Evans and Rothbart, 2007) in a large non-clinical sample $(N=700$,

Table 2

Cronbach's alpha for the three scales across experiments.

\begin{tabular}{lllll}
\hline & Effortful control & Depression & Anxiety & Stress \\
\hline Experiment 1 & .81 & .91 & .83 & .88 \\
Experiment 2 & .79 & .89 & .81 & .88 \\
Experiment 3 & .77 & .96 & .93 & .93 \\
Experiment 4 & .76 & .91 & .77 & .92 \\
Experiment 5 & .83 & .92 & .79 & .86 \\
Experiment 6 & .80 & .89 & .78 & .91 \\
\hline
\end{tabular}

397 females, mean age $=58.7)$ with a mean score of $3.77(\mathrm{SD}=.54)$

A between-subjects one-way ANOVA of the questionnaire scores in the six different samples (tested in Experiments 1-6) yielded no significant differences (all $p>.20)$.

Participants filled out the questionnaires directly before the respective experiment.

\subsection{Experiments}

Two different paradigms were applied in six experiments. Experiments 1-3 utilized a version of the flanker task in which participants identified the ink color of a centrally presented word while ignoring the color of the flanker words above and below the target word. Flanker and target colors could be identical or different, creating congruent and incongruent trials. Words were selected from the Leipzig Affective Norms for German (Kanske and Kotz, 2010a, 2011b, 2012) and were either neutral and negative (Experiments 1 and 3 ) or positive (Experiment 2). This enabled us to study the influence of emotion on the resolution of response conflict in incongruent trials. Experiments 4-6 used an auditory Simon task in which participants identified the gender of a voice presented to the right or left ear and responded with a left or right hand button press. Presentation and response sides could be identical or different, yielding congruent and incongruent trials. The voice recordings were taken from the auditory version of the Leipzig Affective Norms for German (Kanske and Kotz, 2012) and were again neutral and negative (Experiments 4 and 6) or positive words (Experiment 5) spoken with the respective emotional intonation. Again, this procedure allowed us to test conflict resolution in emotional and neutral stimuli. In addition to behavioral responses, we collected EEG (Experiments 1, 2, 4, and 5) and fMRI data (Experiments 3 and 6).

In the EEG, after visual inspection and a time-line analysis, we found a negative deflection that was increased for conflict trials and occurred between 190 and $250 \mathrm{~ms}$ in the visual experiments (Experiments 1 and 2) and between 420 and $550 \mathrm{~ms}$ in the auditory experiments (Experiments 4 and 5). Activations in the fMRI included the anterior cingulate cortex for conflict and the bilateral amygdala for emotion (see Table 3; $p<.05$ with a multiple comparisons correction using Monte-Carlo simulations and a regions of interest analysis for the amygdala). 
Table 3

Talairach coordinates of the peak activations in the ventral ACC and the amygdala in the visual flanker (Experiments 3) and auditory Simon task (Experiment 6). $\mathrm{H}$, hemisphere; Cs, cluster size in cubic millimeters.

\begin{tabular}{lrrrrrr}
\hline & $\mathrm{H}$ & \multicolumn{1}{c}{$x$} & \multicolumn{1}{c}{$y$} & \multicolumn{1}{c}{ Cs } & \multicolumn{1}{c}{$\mathrm{Z}$} \\
\hline Visual flanker task & & & & & & \\
Ventral ACC & $\mathrm{L}$ & -9 & 16 & -9 & 459 & 3.41 \\
& $\mathrm{R}$ & 7 & 19 & -9 & 1026 & 3.57 \\
Amygdala & $\mathrm{L}$ & -16 & -8 & -12 & 135 & 2.61 \\
& $\mathrm{R}$ & 19 & -8 & -12 & 108 & 2.61 \\
Auditory Simon task & & & & & & \\
Ventral ACC & $\mathrm{R}$ & 10 & 33 & 12 & 675 & 3.17 \\
Amygdala & $\mathrm{L}$ & 22 & -9 & -12 & 189 & 2.40 \\
& $\mathrm{R}$ & -20 & -10 & -11 & 180 & 2.61 \\
\hline
\end{tabular}

For further details about samples, stimuli, experimental procedures, and EEG and fMRI data collection, please see the respective publications of the primary data (Kanske and Kotz, 2010b, 2011a,c,d,e).

\subsection{Data analysis}

To obtain a measure for conflict processing speed, we used the reaction time (RT) difference between incongruent and congruent trials, henceforth referred to as conflict score. Note that reduced conflict scores indicate faster conflict resolution. As a measure of the influence of emotion on conflict processing, we used the difference between conflict scores in emotional and neutral trials, referred to as emotion-conflict score. This yields reduced (negative) scores when conflict processing in emotional trials is faster than in neutral trials. We proceeded similarly with the EEG and fMRI data. Thus, we derived an EEG conflict negativity score by subtracting the ERP response to incongruent and congruent trials over anterior electrode sites at which the effect was significant across all EEG experiments (FP1, AF3, AF7, F3, F5, F7, F9, FC3, FC5, FT7, FT9, FP2, AF4, AF8, F4, F6, F8, F10, FC4, FC6, FT8, FT10). Subtracting the conflict negativity score in emotional and neutral trials resulted in an emotion-conflict negativity score indexing the size of the amplitude increase in emotional compared to neutral trials. As the ventral ACC is the target area for emotional conflict processing in fMRI, we correlated the percent signal change in this area with the questionnaire data. Again, we used the difference between the conflict activation (incongruent - congruent) in emotional and neutral trials to quantify the degree to which the ventral ACC was activated more strongly in emotional trials. As depression and anxiety have been related to amygdala activation, we also correlated the activation of the amygdala to emotional stimuli (emotional-neutral) to the emotional state scores (Fig. 1).

\section{Results}

Individual data in effortful control, depression, anxiety, and stress was correlated with performance and neural responses in each experiment (for a complete list, see Table 1). This yielded a largely consistent pattern of relations which is illustrated in Fig. 2.

\subsection{Effortful control}

Across all experiments, effortful control was correlated to reduced conflict scores, i.e., participants scoring high in effortful control were faster in solving flanker (Experiments 1-3) and Simon (Experiments 4 and 5 ) types of conflict (all $p<.05$, except for Experiment 3 , in which the correlation was only marginally significant). This is also reflected in a large increase in the ERP conflict negativity in incongruent trials in participants with high effortful control (all $p<.05$ ). Interestingly, effortful control also correlated significantly with the reduction of RT conflict scores in emotional trials. High effortful control was associated with faster conflict resolution in both negative (Experiments 1, 3, 4 and 6) and positive (Experiments 2 and 5) emotional stimuli. Consequently, the enlarged ERP conflict negativity in emotional trials was stronger in high effortful control participants (all $p<.05$ ). Effortful control was also associated with greater activation of the ventral ACC in emotionally negative trials. There was, however, no correlation with activity in the amygdala.

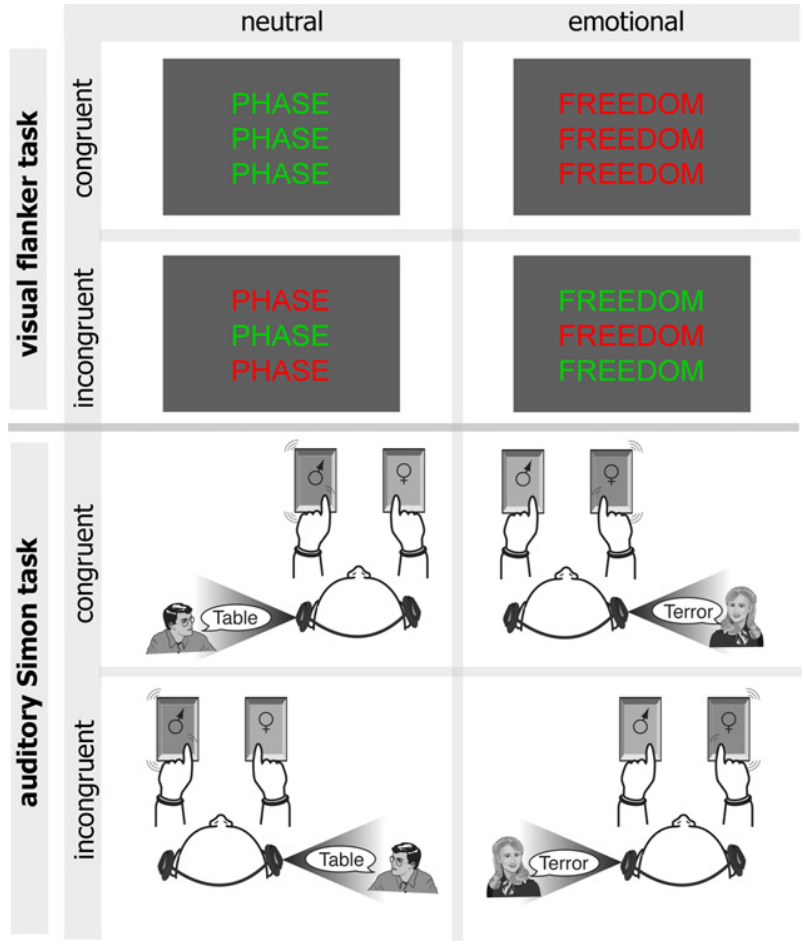

Fig. 1. Schematic of the visual flanker task (Experiments 1-3) and auditory Simon task (Experiments 4-6). In the flanker task, conflict was elicited through incongruent target (center word) and flanker word colors. The Simon task elicited conflict through incongruent stimulus presentation and response sides. Neutral and emotionally negative (Experiments 1, 3, 4, 6) or positive (Experiments 2 and 5) words were presented.

\subsection{Depression, anxiety, and stress}

Depression and anxiety were not related to conflict processing per se. However, we found significant correlations with the influence of emotion on conflict processing. The observed speed in conflict processing in negative emotional trials was reduced in participants scoring high in depression and anxiety. This pattern was observed in all experiments using negative emotional stimuli (Experiments 1, 3, 4 and 6), but not when stimuli were emotionally positive (Experiments 2 and 5). Correspondingly, a normally enlarged ERP conflict negativity in negative emotional trials was reduced in participants with high depression and anxiety scores. Activity increases in the ventral ACC in negative emotional trials for conflict were also negatively related to depression and anxiety. Amygdala activity, in contrast, was higher in participants scoring high in depression and anxiety (all $p<.05$, except for the left amygdala in Experiment 6, which was only marginally significant). There were no significant correlations between the stress scale and any of the measures assessed.

\section{Discussion}

The present data yield new insight into the impact of temperament and subclinical anxiety and depression on the emotional modulation of executive attentional control. Inter-individual differences in temperamental effortful control, anxiety, and depression assessed in questionnaires were consistently associated with the experimental effects. In reaction to emotional stimuli, participants high in effortful control and low in anxiety and depression showed a strong enhancement in attentional control. Also, the ERP conflict negativity was further increased, as was the activation in the ventral ACC. For effortful control, these effects were observed 

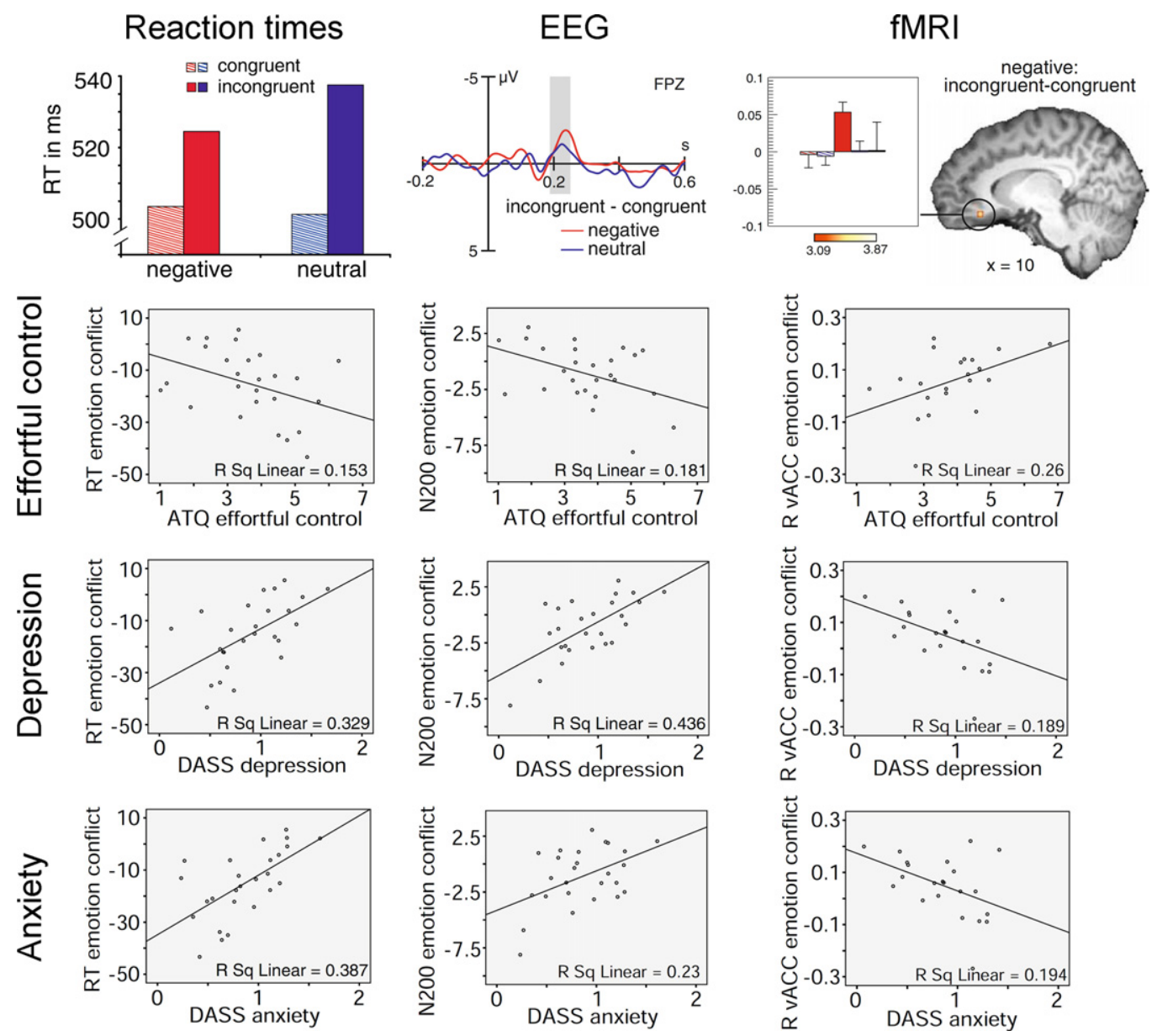

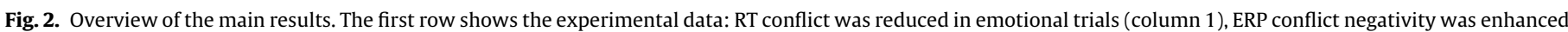

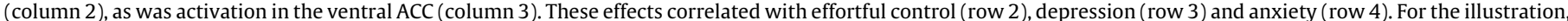

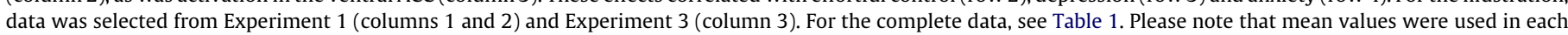
scale and that negative correlations with the ERP measures indicate an increase in amplitude, as the component of interest was a negative deflection.

for both negative and positive emotional stimuli. Depression and anxiety only showed correlations with the effects of negative, but not positive trials.

Effortful control also correlated directly with conflict processing in both flanker and Simon tasks. While this type of result has previously been shown in developmental studies (Gerardi-Caulton, 2000; Rothbart et al., 2003; Rueda et al., 2005), the present results are the first to demonstrate a correlation of effortful control and conflict measures in adults. In line with this correlation in behavioral data, effortful control was also associated with the amplitude of the ERP conflict negativity. Participants high in effortful control showed larger conflict negativities. An increase in the amplitude of this component along with enhanced behavioral conflict resolution has been observed in developmental studies (Ladouceur et al., 2004, 2007; Rueda et al., 2005) and in aging populations (West, 2004), as well as in our own previous studies (Kanske and Kotz, 2010b, 2011a,c,d,e). This suggests that the ERP conflict negativity indexes the amount of resources recruited for conflict processing. Participants high in effortful control seem to have done this more extensively as they solve conflict faster and show larger ERP conflict negativity amplitudes.

Similarly, effortful control was associated with a larger ERP conflict negativity and a large acceleration of conflict resolution in emotional trials. As described above, effortful control is related to executive attention, but also to emotional processing (Carlson and Wang, 2007; Salmon and Pereira, 2002; Spinrad et al., 2007;
Valiente et al., 2007). In particular, it is the regulation of different emotional states including anger and joy (Kochanska et al., 2000) and the appropriate management of emotions to reach certain goals, for example in delayed gratification, that is associated with effortful control (Eisenberg, 2000; Krueger et al., 1996; White et al., 1994). This fits well with the present results. The data show that emotion can modulate executive attentional control, enhancing goal-directed behavior in situations of conflicting response tendencies. In high effortful control participants, this modulatory influence of emotion was increased, suggesting that they better managed and utilized salient emotional information. Interestingly, this effect was accompanied not only by the described ERP effect, but also by enhanced activity in the ventral ACC. This region has been shown to respond sensitively to conflict processing in emotionally negative trials (Kanske and Kotz, 2011c,d). It is also functionally connected to the dorsal ACC and the amygdala in emotionally negative conflict trials, which suggests that it integrates conflict and emotional information signaled by the respective regions. Enhanced activation of the ventral ACC seems to underlie the observed behavioral facilitation of conflict processing in emotional trials. It, thus, constitutes an emotion specific brain mechanism of executive attention, in contrast to non-emotional attention mechanisms that do not involve the ventral ACC. Because of its emotion specificity, it is plausible to find influences of individual variations in depression and anxiety.

Depression and anxiety yielded a correlational pattern that was largely opposite to that of effortful control. Participants high 
in depression and anxiety showed reduced enhancement of conflict processing in negative emotional stimuli. Consequently, the expected amplitude increase of the conflict negativity and the enhanced activation of the ventral ACC activation were reduced. This is in line with previous work in clinical (Bradley et al., 1999; Gerdes et al., 2008; Mitterschiffthaler et al., 2008; Mühlberger et al., 2006) and subclinical depression and anxiety (Li et al., 2008; Mercado et al., 2006; Scott et al., 2001), which showed altered processing of negative emotional stimuli. Among other indices, this was shown by a heightened sensitivity for negative emotional stimuli (Bar-Haim et al., 2007; Fox et al., 2002; Mogg et al., 2007), as well as hyperactivation in the amygdala and deficient ACC activity (Drevets et al., 1997; Siegle et al., 2007). Interestingly, the cognitive regulation of negative emotion and amygdala activation is also distorted in depression (Johnstone et al., 2007). As effortful control has been shown to be related to emotion regulation capabilities (Carlson and Wang, 2007; Kochanska et al., 2000; Salmon and Pereira, 2002; Spinrad et al., 2007; Valiente et al., 2007), it is possible that effortful control influences the relation of depression and anxiety to the emotional modulation of attentional control. It would suggest that enhancing emotion regulation skills in participants scoring high in depression and anxiety, possibly through psychotherapeutic training, could have a beneficial effect and help to overcome the dysfunctional pattern observed in these individual.

Just as the behavioral effects were different for effortful control vs. depression and anxiety, so too were the ERP and fMRI data. High depression and anxiety resulted in a reduced increase of the conflict negativity and of ventral ACC activity. Amygdala activation, in contrast, was enhanced. The increased sensitivity toward negative stimuli and the deficit in negative emotion regulation suggest that depression and anxiety yield a less appropriate evaluation of emotionally negative stimuli. Consequently, the benefit in resolving conflict in emotional trials is reduced.

The correlations of depression and anxiety with RT and ERP data (note that there was no positive emotional condition in the fMRI study) were specific to negative emotion. The influence of positive emotional stimuli on conflict processing was not related to depression and anxiety. This is in line with previous evidence that failed to show a consistent deviation of processing positive emotional stimuli in depression (Gotlib and Hamilton, 2008) and also in anxiety (Etkin and Wager, 2007).

There were no significant correlations between individual stress level and any of the experimental measures. Even though stress has been shown to increase amygdala responses to emotional stimuli (van Marle et al., 2009) and also to slow performance when emotional distracters are presented (Oei et al., 2012), it is still unclear if these effects are only present when stress is experimentally induced as has been done in these studies, or if they generalize to more chronic stress levels over longer amounts of time measured with questionnaires. The lack of significant correlations in the present data may provide some indication for no effect of this type of stress, but future studies should specifically address this point. The diverging results for stress, anxiety, and depression despite their conceptual relation, however, support the specificity of the correlations for depression and anxiety.

The validity of the present results is supported by the fact that correlational patterns were replicated in each experiment. Even though the sample size in a single experiment may have been rather small, the entire sample (across experiments: $N=142$ ) is adequate for a correlation study. It may seem suggestive to simply pool (at least the behavioral) data from all participants; however, this is quite problematic due to the differences in the stimuli (positive and negative; visual and auditory), the experimental design (Simon and flanker tasks), and the setting (EEG lab and fMRI scanner). This, of course, led to a large number of tests. Nevertheless, a correction of multiple comparisons is inappropriate as described by Perneger
(1998). Testing the whole pattern of correlations between the questionnaire and the RT, ERP, and fMRI data provides more and very specific evidence. Also, repetitively testing a correlational pattern in each experiment is a very rigorous test as it provides multiple possibilities to reject our hypotheses (see also Hensch et al., 2007). It is, therefore, a strong point of the present results that the correlational pattern was replicated in two different conflict tasks, the flanker (Eriksen and Eriksen, 1974) and the Simon paradigm (Simon and Rudell, 1967). Also, the effects were independent of the specific sensory modality as visual and auditory stimulus presentation yielded largely comparable results.

In addition to varying the methods used to measure executive attentional control, future studies could also use multiple operationalizations for depression and anxiety, as there are a number of different valid questionnaires available. This may also help to disentangle the effects of depression and anxiety. In the present data, these effects are very similar, mainly due to the high correlation between the two scales. They amount to .50 as reported in the manual (Lovibond and Lovibond, 1995b), despite the original aim of the authors to clearly separate depression and anxiety. Another possibility would be to use more specific emotional stimuli. The negative words used in the present experiments were not differentiated regarding primary emotional categories, which may be more sensitive to either anxiety (fear-eliciting stimuli) or depression (sadness-related stimuli). A third possibility to address this point would be to investigate patient populations. These could differentiate depression and anxiety much more clearly than the present student populations could.

Application of the paradigm to patient populations would also be interesting for a number of other reasons. First, this could validate the correlational pattern, which is currently based on relatively small subclinical variations in anxiety and depression. Relatively low scores on dysfunctional thoughts and behavior might not be very meaningful, which could also influence the correlations of these scores with other measures. Testing patient populations and matched healthy controls would resolve this issue. Second, the design developed for the present experiments may prove useful to investigate deficient emotional processing in patient groups. Third, effortful control showed the opposite relation to the influence of emotion on executive attention than depression and anxiety. As effortful control describes the ability to regulate attentional processes and is associated with emotion regulation, this may indicate that individuals high in depression or anxiety could benefit from training in these abilities.

To conclude, the present results contribute to our understanding of the interaction of emotion and executive attentional control on multiple levels. Across visual and auditory modalities, across positive and negative emotional stimuli, and across Simon and flanker type of conflict tasks, emotion enhances attentional control by increased activation of neural resources involved in conflict resolution. Combining data from these diverse sources strengthens the conclusions drawn and allows some generalization of the effects. Using a correlational approach, we could show that individual variations in effortful control, anxiety, and depression are related to this effect across the different methodological permutations. This data is important as it suggests that emotion attention interactions are subject to change due to the emotional state of an individual and its self-regulatory competencies.

\section{Acknowledgement}

This work was supported by the German Research Council(DFG) [DFG-Graduate Program [1182]: Function of Attention in Cognition, University of Leipzig, Germany, DFG-FOR-499 to SAK, Max 
Planck Institute for Human Cognitive and Brain Sciences, Leipzig, Germany].

\section{References}

Abler, B., Erk, S., Herwig, U., Walter, H., 2007. Anticipation of aversive stimuli activates extended amygdala in unipolar depression. Journal of Psychiatric Research 41, 511-522.

Bar-Haim, Y., Lamy, D., Glickman, S., 2005. Attentional bias in anxiety: a behavioral and ERP study. Brain and Cognition 59, 11-22.

Bar-Haim, Y., Lamy, D., Pergamin, L., Bakermans-Kranenburg, M.J., Jzendoorn van, I.M.H., 2007. Threat-related attentional bias in anxious and nonanxious individuals: a meta-analytic study. Psychological Bulletin 133, 1-24.

Bishop, S.J., 2007. Neurocognitive mechanisms of anxiety: an integrative account. Trends in Cognitive Sciences 11, 307-316.

Bishop, S.J., Duncan, J., Lawrence, A.D., 2004. State anxiety modulation of the amygdala response to unattended threat-related stimuli. Journal of Neuroscience 24, 10364-10368.

Blasi, G., Mattay, V.S., Bertolino, A., Elvevåg, B., Callicott, J.H., Das, S., Kolachana, B.S., Egan, M.F., Goldberg, T.E., Weinberger, D.R., 2005. Effect of catecholO-methyltransferase val158met genotype on attentional control. Journal of Neuroscience 25, 5038-5045.

Bradley, B.P., Mogg, K., White, J., Groom, C., de Bono, J., 1999. Attentional bias for emotional faces in generalized anxiety disorder. British Journal of Clinical Psychology 38, 267-278.

Carlson, S.M., Wang, T.S., 2007. Inhibitory control and emotion regulation in preschool children. Cognitive Development 22, 489-510.

Diamond, A., Briand, L., Fossella, J., Gehlbach, L., 2004. Genetic and neurochemical modulation of prefrontal cognitive functions in children. American Journal of Psychiatry 161, 125-132.

Drevets, W.C., 2001. Neuroimaging and neuropathological studies of depression: implications for the cognitive-emotional features of mood disorders. Current Opinion in Neurobiology 11, 240-249.

Drevets, W.C., Price, J.L., Simpson, J.R., Todd, R.D., Reich, T., Vannier, M., Raichle, M.E., 1997. Subgenual prefrontal cortex abnormalities in mood disorders. Nature 386, 824-827.

Eisenberg, N., 2000. Emotion, regulation, and moral development. Annual Review of Psychology 51, 665-697.

Eriksen, B.A., Eriksen, C.W., 1974. Effects of noise letters upon the identification of a target letter in a nonsearch task. Perception and Psychophysics 16, 143-149.

Etkin, A., Wager, T.D., 2007. Functional neuroimaging of anxiety: a meta-analysis of emotional processing in PTSD, social anxiety disorder, and specific phobia. American Journal of Psychiatry 164, 1476-1488.

Evans, D., Rothbart, M.K., 2007. Developing a model for adult temperament. Journal of Research in Personality 41, 868-888.

Fan, J., Fossella, J., Sommer, T., Wu, Y., Posner, M.I., 2003. Mapping the genetic variation of executive attention onto brain activity. Proceedings of the National Academy of Sciences of the United States of America 100, 7406-7411.

Fan, J., Wu, Y., Fossella, J.A., Posner, M.I., 2001. Assessing the heritability of attentional networks. BMC Neuroscience 2, 14

Folstein, Van Petten, 2008. Influence of cognitive control and mismatch on the N2 component of the ERP: A review. Psychophysiology 45, 152-170.

Fossella, J., Sommer, T., Fan, J., Wu, Y., Swanson, J.M., Pfaff, D.W., Posner, M.I., 2002. Assessing the molecular genetics of attention networks. BMC Neuroscience 3, 14.

Fox, E., Russo, R., Bowles, R., Dutton, K., 2001. Do threatening stimuli draw or hold visual attention in subclinical anxiety? Journal of Experimental Psychology: General 130, 681-700.

Fox, E., Russo, R., Dutton, K., 2002. Attentional bias for threat: evidence for delayed disengagement from emotional faces. Cognition and Emotion 16, 355-379.

Garner, M., Mogg, K., Bradley, B.P., 2006. Orienting and maintenance of gaze to facial expressions in social anxiety. Journal of Abnormal Psychology 115, 760-770.

Gerardi-Caulton, G., 2000. Sensitivity to spatial conflict and the development of selfregulation in children 24-36 months of age. Developmental Science 3, 397-404.

Gerdes, A.B.M., Alpers, G.W., Pauli, P., 2008. When spiders appear suddenly: spiderphobic patients are distracted by task-irrelevant spiders. Behaviour Research and Therapy 46, 174-187.

Goldsmith, H.H., Lemery, K.S., Buss, K.A., Campos, J.J., 1999. Genetic analyses of focal aspects of infant temperament. Developmental Psychology 35, 972-985.

Gotlib, I.H., Hamilton, J.P., 2008. Neuroimaging and depression: current status and unresolved issues. Current Directions in Psychological Science 17, 159-163.

Hensch, T., Herold, U., Brocke, B., 2007. An electrophysiological endophenotype of hypomanic and hyperthymic personality. Journal of Affective Disorders 101, $13-26$.

Johnstone, T., van Reekum, C.M., Urry, H.L., Kalin, N.H., Davidson, R.J., 2007. Failure to regulate: counterproductive recruitment of top-down prefrontal-subcortical circuitry in major depression. Journal of Neuroscience 27, 8877-8884.

Kalia, M., 2005. Neurobiological basis of depression: an update. Metabolism: Clinical and Experimental 54, 24-27.

Kanske, P., Kotz, S.A., 2010a. Leipzig Affective Norms for German: a reliability study. Behavior Research Methods 42, 987-991.

Kanske, P., Kotz, S.A., 2010b. Modulation of early conflict processing: N200 responses to emotional words in a flanker task. Neuropsychologia 48, 3661-3664.

Kanske, P., Kotz, S.A., 2011a. Conflict processing is modulated by positive emotion: ERP data from a flanker task. Behavioural Brain Research 219, 382-386.
Kanske, P., Kotz, S.A., 2011b. Cross-modal validation of the Leipzig Affective Norms for German (LANG). Behavior Research Methods 43, 409-413.

Kanske, P., Kotz, S.A., 2011c. Emotion speeds up conflict resolution: a new role for the ventral anterior cingulate cortex? Cerebral Cortex 21, 911-919.

Kanske, P., Kotz, S.A., 2011d. Emotion triggers executive attention: anterior cingulate cortex and amygdala responses to emotional words in a conflict task. Human Brain Mapping 32, 198-208.

Kanske, P., Kotz, S.A., 2011e. Positive emotion speeds up conflict processing: ERP responses in an auditory Simon task. Biological Psychology 87, 122-127.

Kanske, P., Kotz, S.A., 2012. Auditory affective norms for German: testing the influence of depression and anxiety on valence and arousal ratings. PLoS One 7 e30086.

Kochanska, G., 1997. Multiple pathways to conscience for children with different temperaments: from toddlerhood to age 5. Developmental Psychology 33 228-240.

Kochanska, G., Murray, K.T., Harlan, E.T., 2000. Effortful control in early childhood: continuity and change, antecedents, and implications for social development. Developmental Psychology 36, 220-232.

Krueger, R.F., Caspi, A., Moffitt, T.E., White, J., Stouthamer-Loeber, M., 1996. Delay of gratification, psychopathology, and personality: is low self-control specific to externalizing problems? Journal of Personality 64, 107-129.

Ladouceur, C.D., Dahl, R.E., Carter, C.S., 2004. ERP correlates of action monitoring in adolescence. Annals of the New York Academy of Sciences 1021 329-336.

Ladouceur, C.D., Dahl, R.E., Carter, C.S., 2007. Development of action monitoring through adolescence into adulthood: ERP and source localization. Developmental science $10,874-891$.

Lee, B.-T., Cho, S.W., Khang, H.S., Lee, B.-C., Choi, I.-G., Lyoo, I.K., Ham, B.-J., 2007. The neural substrates of affective processing toward positive and negative affective pictures in patients with major depressive disorder. Progress in NeuroPsychopharmacology and Biological Psychiatry 31, 1487-1492.

Li, W., Paller, K.A., Zinbarg, R.E., 2008. Conscious intrusion of threat information via unconscious priming in anxiety. Cognition and Emotion 22, 44-62.

Li, W., Zinbarg, R.E., Paller, K.A., 2007. Trait anxiety modulates supraliminal and subliminal threat: brain potential evidence for early and late processing influences. Cognitive, Affective and Behavioral Neuroscience 7, 25-36.

Lovibond, P.F., Lovibond, S.H., 1995a. The structure of negative emotional states comparison of the depression anxiety stress scales (DASS) with the beck depression and anxiety inventories. Behaviour Research and Therapy 33, 335-343.

Lovibond, S.H., Lovibond, P.F., 1995b. Manual for the depression anxiety stress scales 2nd ed. Psychology Foundation, Sydney.

Mercado, F., Carretié, L., Tapia, M., Gómez-Jarabo, G., 2006. The influence of emotional context on attention in anxious subjects: neurophysiological correlates. Journal of Anxiety Disorders 20, 72-84.

Mitterschiffthaler, M.T., Williams, S.C.R., Walsh, N.D., Cleare, A.J., Donaldson, C., Scott, J., Fu, C.H.Y., 2008. Neural basis of the emotional Stroop interference effect in major depression. Psychological Medicine 38, 247-256.

Mogg, K., Bradley, B.P., 1999. Some methodological issues in assessing attentiona biases for threatening faces in anxiety: a replication study using a modified version of the probe detection task. Behaviour Research and Therapy 37 595-604.

Mogg, K., Garner, M., Bradley, B.P., 2007. Anxiety and orienting of gaze to angry and fearful faces. Biological Psychology 76, 163-169.

Mogg, K., Millar, N., Bradley, B.P., 2000. Biases in eye movements to threatening facial expressions in generalized anxiety disorder and depressive disorder. Journal of Abnormal Psychology 109, 695-704.

Mogg, K., Philippot, P., Bradley, B.P., 2004. Selective attention to angry faces in clinical social phobia. Journal of Abnormal Psychology 113, 160-165.

Moriya, J., Tanno, Y., 2008. Relationships between negative emotionality and attentional control in effortful control. Personality and Individual Differences 44, 1348-1355.

Most, S.B., Chun, M.M., Johnson, M.R., Kiehl, K.A., 2006. Attentional modulation of the amygdala varies with personality. Neuroimage 31, 934-944.

Mühlberger, A., Wiedemann, G., Herrmann, M.J., Pauli, P., 2006. Phylo- and ontogenetic fears and the expectation of danger: differences between spider- and flight-phobic subjects in cognitive and physiological responses to disorderspecific stimuli. Journal of Abnormal Psychology 115, 580-589.

Norman, D.A., Shallice, T., 1986. Attention to action: willed and automatic control of behavior. In: Davidson, R.J., Schwartz, G.E., Shapiro, D. (Eds.), Consciousness and Self-regulation. Plenum Press, New York, pp. 1-18.

Oei, N.Y., Veer, I.M., Wolf, O.T., Spinhoven, P., Rombouts, S.A., Elzinga, B.M., 2012 Stress shifts brain activation towards ventral 'affective' areas during emotional distraction. Social Cognitive and Affective Neuroscience 7, 403-412.

Oldfield, R.C., 1971. The assessment and analysis of handedness: the Edinburgh inventory. Neuropsychologia 9, 97-98.

Perneger, T.V., 1998. What's wrong with Bonferroni adjustments. BMJ Clinica Research 316, 1236-1238.

Pezawas, L., Meyer-Lindenberg, A., Drabant, E.M., Verchinski, B.A., Munoz, K.E. Kolachana, B.S., Egan, M.F., Mattay, V.S., Hariri, A.R., Weinberger, D.R., 2005. 5-HTTLPR polymorphism impacts human cingulate-amygdala interactions: a genetic susceptibility mechanism for depression. Nature Neuroscience 8 828-834

Rothbart, M.K., 2007. Temperament, development, and personality. Current Directions in Psychological Science 16, 207-212.

Rothbart, M.K., Ahadi, S.A., Evans, D.E., 2000. Temperament and personality: origins and outcomes. Journal of Personality and Social Psychology 78, 122-135. 
Rothbart, M.K., Bates, J.E., 2006. Temperament. In: Damon, W., Lerner, R., Eisenberg, N. (Eds.), Handbook of Child Psychology: Social, Emotional, and Personality Development, vol. 3, 6th ed. Wiley, New York, pp. 99-166.

Rothbart, M.K., Ellis, L.K., Rueda, M.R., Posner, M.I., 2003. Developing mechanisms of temperamental effortful control. Journal of Personality 71, 1113-1143.

Rueda, M.R., Rothbart, M.K., McCandliss, B.D., Saccomanno, L., Posner, M.I., 2005 Training, maturation, and genetic influences on the development of executive attention. Proceedings of the National Academy of Sciences of the United States of America 102, 14931-14936.

Salmon, K., Pereira, J.K., 2002. Predicting children's response to an invasive medical investigation: the Influence of effortful control and parent behavior. Journal of Pediatric Psychology 27, 227-233.

Scott, K.M., Mogg, K., Bradley, B.P., 2001. Masked semantic priming of emotional information in subclinical depression. Cognitive Therapy and Research 25, 505-524.

Siegle, G.J., Thompson, W., Carter, C.S., Steinhauer, S.R., Thase, M.E., 2007. Increased amygdala and decreased dorsolateral prefrontal BOLD responses in unipolar depression: related and independent features. Biological Psychiatry 61, 198-209.
Simon, J.R., Rudell, A.P., 1967. Auditory S-R compatibility: the effect of an irrelevant cue on information processing. Journal of Applied Psychology 51, 300-304.

Spinrad, T.L., Eisenberg, N., Gaertner, B.M., 2007. Measures of effortful regulation for young children. Infant Mental Health Journal 28, 606-626.

Valiente, C., Lemery-Chalfant, K., Reiser, M., 2007. Pathways to problem behaviors: chaotic homes, parent and child effortful control, and parenting. Social Development 16, 249-267.

van Marle, H.J.F., Hermans, E.J., Qin, S., Fernández, G., 2009. From specificity to sensitivity: how acute stress affects amygdala processing of biologically salient stimuli. Biological Psychiatry 66, 649-655.

Weinstein, A.M., 1995. Visual ERPs evidence for enhanced processing of threatening information in anxious university students. Biological Psychiatry 37, 847-858.

West, R., 2004. The effects of aging on controlled attention and conflict processing in the Stroop task. Journal of Cognitive Neuroscience 16, 103-113.

White, J.L., Moffitt, T.E., Caspi, A., Bartusch, D.J., Needles, D.J., Stouthamer-Loeber, M., 1994. Measuring impulsivity and examining its relationship to delinquency. Journal of Abnormal Psychology 103, 192-205. 